\title{
L-Arginine alleviates heat stress-induced intestinal epithelial barrier damage by promoting expression of tight junction proteins via the AMPK pathway
}

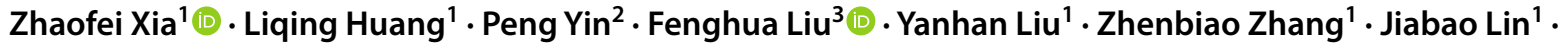 \\ Wenshu Zou ${ }^{3} \cdot$ Chunxiao $\mathrm{Li}^{3}$
}

Received: 14 June 2019 / Accepted: 22 September 2019 / Published online: 1 October 2019

(c) The Author(s) 2019

\begin{abstract}
Heat stress (HS) and secondary restricted blood flow to the intestines cause dysfunction of the intestinal epithelial barrier. Tight junctions (TJs) are essential to maintain intestinal integrity. L-Arginine has beneficial effects on gut functions. However, the underlying mechanisms remain largely unknown. This study tested the hypothesis that L-arginine regulates the TJ network by activating AMP-activated protein kinase (AMPK) signaling, which in turn improves intestinal barrier functions under HS. IEC-6 cells and rat small intestines were used as experiment models of heat stress. AICAR and dorsomorphin were used to activate and inhibit the AMPK pathway, respectively. Cell proliferation, apoptosis, differential gene expression and KEGG pathway analysis, intestinal paracellular permeability, intestinal morphology, and expression of HSP and TJ proteins, and p-AMPK were determined. L-Arginine promoted cell proliferation and reduced apoptosis after heat exposure at an optimal concentration of $5 \mathrm{mmol}$. Transcriptome sequencing analysis revealed that differentially expressed genes associated with the HSP family and TJs were elevated by L-arginine. According to KEGG pathway analysis, L-arginine activated the AMPK signaling pathway. In vivo, intestinal damage resulted in obvious morphological changes as well as apoptosis with TUNEL and caspase-3 staining under HS and dorsomorphin treatments. Furthermore, HS and dorsomorphin increased the serum D-lactate concentration, diamine oxidase activity, and mRNA expression level of MLCK $(P<0.05)$. In contrast, L-arginine and AICAR treatments reduced intestinal injury, maintained intestinal permeability, and increased the villus/crypt ratio under hyperthermia. L-Arginine had the same effect as AICAR both in vitro and in vivo, namely increasing p-AMPK protein expression. L-Arginine and AICAR also upregulated the mRNA expression level of HSP70 and HSP90, and downregulated mRNA expression of MLCK $(P<0.05)$. The protein expression levels of TJ proteins ZO-1 and claudin-1 were suppressed by heat stroke and dorsomorphin, but enhanced by L-arginine and AICAR. Our findings indicate that activation of AMPK signaling by L-arginine is associated with improved intestinal mucosal barrier functions by enhancing the expression of TJs in rat small intestines and IEC-6 cells during HS.
\end{abstract}

Keywords Heat stress $\cdot$ L-Arginine $\cdot$ IEC6 cells $\cdot$ Intestines $\cdot$ Tight junctions $\cdot$ AMPK pathway

$\begin{array}{ll}\text { Abbreviations } \\ \text { HS } & \text { Heat stress } \\ \text { HSPs } & \text { Heat shock proteins } \\ \text { HSF } & \text { Heat shock factor } \\ \text { TJs } & \text { Tight junctions }\end{array}$

Electronic supplementary material The online version of this article (https://doi.org/10.1007/s11033-019-05090-1) contains supplementary material, which is available to authorized users.

Zhaofei Xia

drxiacau@163.com

Extended author information available on the last page of the article
ZO Zona occludens

AMPK Adenosine monophosphate-activated protein kinase

LKB1 Liver kinase B1

CaMKK $\mathrm{Ca}^{2+} /$ calmodulin-dependent kinase kinase

\section{Introduction}

As global warming worsens, high ambient temperatures, strenuous activity, or their combination can lead to heat stress (HS) that negatively affects animal performance, health, and livestock value [1]. The gastrointestinal system 
plays key roles in protection absent pathogens, absorption of nutrients, and immunity [2,3]. However, the intestinal barrier is easily subjected to hyperthermia due to reduced blood flood to mesenteric circulation, which can negatively affect the integrity of the intestinal epithelium [4]. Accumulating in vitro and in vivo investigations have demonstrated that HS can weaken intestinal integrity and compromise its barrier function $[5,6]$.

When encountering thermal stress, synthesis of a group of highly conserved proteins commonly known as heat shock proteins (HSPs) is enhanced via the phosphorylation of heat shock factors (HSFs) [7]. HSPs are vital for the survival of stressed cells and stabilization of hemostasis. In general, HSPs are important for the quality of synthesized proteins by preventing the misfolding of proteins and degrading damaged proteins [8]. In addition, they are involved in multiple physiological processes including cell proliferation, apoptosis, and stress responses [9]. According to their molecular size, HSPs are classified into main six families, namely small HSPs, HSP40, HSP60, HSP70, HSP90, and HSP100 [10]. HSP70 and HSP90 are the most broadly studied proteins among the HSP families [11]. Hao et al., found that heat stress increases HSP70 expression in the small intestine [12]. Uerlings et al. demonstrated that gene expression of HSF1, HSP70, and HSP90 is elevated under HS [13].

Intestinal epithelial cells are connected through apical junctions containing tight junctions (TJs) and adherens junctions [14]. Theses apical junctions seal the space between enterocytes and maintain the integrity and tightness of the intestinal mucosal barrier [15]. Claudins, occludin, and junctional adhesion molecules are primary constituents of TJs that are stabilized by the intracellular scaffolding protein zona occludens (ZO) [16]. Dysfunction of TJs has been reported to be associated with increased permeability of the intestinal epithelium [17] and the development and progression of intestinal disorders [18]. HS decreases the expression of TJs and leads to intestinal barrier dysfunction [19]. Zhang et al. also reported that heat stress disrupts the expression of TJ proteins in chickens, such as mucin-2, claudin-1, and occludin [20]. Adenosine monophosphate-activated protein kinase (AMPK), a highly conserved serine-threonine kinase, acts as a central metabolic regulator and plays a key role in energy balance. AMPK activity is triggered by energy depletion, such as heat shock, ischemia, oxidative stress, hypoxia, and exercise [21]. Two major upstream AMPK regulatory kinases have been discovered, liver kinase B1 (LKB1) and $\mathrm{Ca}^{2+} /$ calmodulin-dependent kinase kinase (CaMKK). LKB1 activates AMPK following an increase of the AMP/ATP ratio [22], whereas CaMKK phosphorylates AMPK depending on the intracellular concentration of $\mathrm{Ca}^{2+}$ [23]. AMPK phosphorylation is reported to be involved in tight junction assembly and cell polarization [21]. Activation of AMPK by metformin maintains TJs in the colonic epithelium of mice and significantly controls the progression of colitis [24]. AMPK activation promotes TJs assembly in Caco-3 cells by enhancing ZO-1 and occludin expression [25].

L-Arginine has a positive effect on the intestinal barrier function. Dietary L-arginine supplementation reduces intestinal mucosal damage caused by methotrexate in rats [26]. L-Arginine is effective to maintain intestinal permeability in mice subjected to physical exercise under environmental HS [27]. Considering the deleterious effect of heat stress on tight junction proteins and previous investigations indicating the protective effect of L-arginine on the intestinal barrier, we assessed whether L-arginine affects the expression of TJ proteins and intestinal integrity by activating the AMPK signaling pathway under heat stress using IEC-6 cells and rat intestines. AICRA (AMPK activator) and dorsomorphin (AMPK inhibitor) were also applied to examine the role of L-arginine in activation of the AMPK pathway both in vitro and in vivo.

\section{Materials and methods}

\section{Cell culture and treatments}

IEC-6 cells (China Infrastructure of Cell Line Resource, Beijing, China) were cultured in Dulbecco's modified Eagle's medium (DMEM) (8112040, Gibco, Carlsbad, CA, USA) supplemented with $10 \%$ fetal bovine serum (10099-141, Gibco), $100 \mathrm{U} / \mathrm{mL}$ penicillin, and $100 \mu \mathrm{g} / \mathrm{mL}$ streptomycin (Gibco, Invitrogen, Carlsbad, CA, USA) at $37{ }^{\circ} \mathrm{C}$ with $5 \%$ $\mathrm{CO}_{2}$. Various non-cytotoxic concentrations of L-arginine (3, 5, 7, and $10 \mathrm{mM}$ ) (A8094, Sigma, St. Louis, MO, USA) were added to the cell culture medium. For heat stress, after $48 \mathrm{~h}$ of incubation, IEC- 6 cells in HS and ARG groups were exposed to $42{ }^{\circ} \mathrm{C}$ with $5 \% \mathrm{CO}_{2}$ for $1.5 \mathrm{~h}$.

For AMPK activation and inhibition experiments, $0.8 \mathrm{mM}$ AICAR (13417, MCE, NJ, USA) or $5 \mu \mathrm{M}$ dorsomorphin dihydrochloride (13418, MCE) were applied to IEC-6 cells at $70-80 \%$ confluence. Then, the IEC- 6 cells were incubated for $24 \mathrm{~h}$ without changing the medium. IEC- 6 cells were then subjected to heat stress at $42{ }^{\circ} \mathrm{C}$ with $5 \% \mathrm{CO}_{2}$ for $1.5 \mathrm{~h}$.

\section{Cell viability assay}

The viability of IEC- 6 cells was determined using a standard MTT assay (4890, R\&D Systems, Minneapolis, MN, USA). Cells were cultured in 96-well plates with DMEM alone or with various concentrations of L-arginine and then treated with $10 \mu \mathrm{L}$ MTT reagent. For the heat stress treatment, specific groups of cells were exposed to HS for $1.5 \mathrm{~h}$. Then, $100 \mu \mathrm{L}$ detergent reagent was added to each well. Absorbance was then measured at $550 \mathrm{~nm}$ (IMark, Bio-Rad, CA, USA). 


\section{Transcriptome sequencing}

Cells were harvested in Trizol (Invitrogen, CA, USA). Total RNA was extracted following the manufacturer's protocol. RNA degradation and contamination were monitored on $1 \%$ agarose gels, and purity was checked with a Nanophotometer spectrophotometer (IMPLEN, CA, USA). For transcriptome sequencing, $3 \mu \mathrm{g}$ RNA per sample was used as input material for the RNA sample preparations. Sequencing libraries were generated using an NEBNext ${ }^{\circledR}$ UltraTM RNA Library Prep Kit for Illumina ${ }^{(N E B, ~ U S A), ~}$ following the manufacturer's recommendations. Index codes were added to attribute sequences to each sample. Then, the library preparations were sequenced on an Illumina Hiseq platform, and 150 bp paired-end reads were generated. Differential expression analysis of two groups was performed using DESeq2 R package (1.16.1). Genes with an adjusted $P$ value of less than 0.05 found by DESeq2 were assigned as differentially expressed. A heatmap was generated using TBtools [28]. ClusterProfiler R package was used to test the statistical enrichment of differentially expressed genes in KEGG pathways.

\section{Animal experiments}

All experimental procedures and protocols were approved by the Animal Care and Use Committee of China Agricultural University (Permit Number: 20181109-6). Forty healthy male Sprague-Dawley rats weighting $200 \pm 20$ g were obtained from Vital River Experimental Animal Company

Table 1 Primers for real-time quantitative PCR assay

\begin{tabular}{lll}
\hline Gene & Primer sequence* $\left(5^{\prime}-3^{\prime}\right)$ & $\begin{array}{l}\text { Product } \\
\text { size } \\
(\mathrm{bp})\end{array}$ \\
\hline MLCK & F: GCTGCCTCTCATCATCAATACG & 128 \\
& R: GCCTGGATTCTGCTTCTGTG & \\
Hsp70 & F: GAAGATGAAGGAGATCGCTGAG & 90 \\
& R: CGCTGAGAGTCGTTGAAGTAG & \\
Hsp90 & F: GTCCCGGTGCGGTAGTCACG & 70 \\
& R: TTGGGTCTGGGTTCCTCAGGC & \\
HSF1 & F: AACGTCCCGGCCTTCCTAA & 67 \\
& R: AGATGAGCGCGTCTGTGTC & \\
ZO-1 & F: GATGGTGCTACAAGTGATG & 92 \\
& R: TCCGTGCTATACATTGAGT & \\
Claudin-1 & F: TGTCCACCATTGGCATGAAG & 220 \\
& R: GCCACTAATGTCGCCAGACC & \\
LKB1 & F: GGTTCTCCATCCGACAGATTAG & 175 \\
& R: TCTTCCTCCTCCTCCTCCTC & \\
$\beta$-actin & F: GGAGATTACTGCCCTGGCTCCTA & 150 \\
& R: GACTCATCGTACTCCTGCTTGCTG & \\
\hline
\end{tabular}

(Beijing, China). Rats were acclimated in a temperaturecontrolled room at $25{ }^{\circ} \mathrm{C}$ with $60 \%$ relative humidity $(\mathrm{RH})$ for 1 week with free access to water and food.

\section{Grouping and sample collection}

After 7 days of acclimation, the rats were randomly divided into five groups with eight in each. Treatments were as follows: control (C), heat stress (HS), heat stress + L-arginine (ARG), heat stress + AICAR (AR), and heat stress + dorsomorphin dihydrochloride (DD). HS, ARG, AR, and DD rats were housed in the same environment as the $\mathrm{C}$ group $\left(25^{\circ} \mathrm{C}\right.$, $60 \% \mathrm{RH})$, but were subjected to heat stress at $40{ }^{\circ} \mathrm{C}$ and $60 \%$ RH from 11:00 am to 1:00 pm daily for 3 consecutive days [11]. Rats in the ARG group were administered daily with a $1 \mathrm{~mL}$ L-arginine solution $(250 \mathrm{mg} / \mathrm{kg}$ body weight $)$ via intragastric gavage at 7 days before the HS treatment until the end of the study period. The rats in AR and DD groups were administered daily with $1 \mathrm{~mL}$ AICAR $(200 \mathrm{mg} / \mathrm{kg})$ and dorsomorphin dihydrochloride $(30 \mathrm{mg} / \mathrm{kg})$, respectively, at 3 days before heat stress. The other two groups were administered with the same dose of saline. Eight rats from each group were sacrificed by decapitation immediately after the stress period.

Rectal temperature was recorded with a digital thermometer right after daily heat exposure. Blood was immediately collected from the heart after sacrifice and centrifuged at $3000 \times g\left(10 \mathrm{~min}, 4^{\circ} \mathrm{C}\right)$ to separate serum. The serum was stored at $-20^{\circ} \mathrm{C}$ until analysis. Jejunum samples were collected for histology as well as mRNA and protein expression analyses. All samples for mRNA and protein analyses were immediately frozen in liquid nitrogen.

\section{Measurement of blood constituents}

The serum cortisol concentration was measured by a chemiluminescence Apparatus (Cobas 2601, Roche, Basel, Switzerland) using a commercial kit (11875116, Roche). The serum D-lactate concentration was assessed by a commercial ELISA kit (Nanjing Jiancheng Bioengineering Institute, Jiangsu, China), according to the manufacturer's instructions. Diamine oxidase activity was measured spectrophotometrically with a commercial kit (Nanjing Jiancheng Bioengineering Institute), following the manufacturer's instructions.

\section{Morphological examination}

To observe pathological changes, jejunum tissues were fixed in $4 \%$ paraformaldehyde for $24 \mathrm{~h}$ and then embedded in paraffin. Sections were stained with hematoxylin and eosin. Nine crypt-villus units were measured in each tissue cross-section. Then, the villus height/crypt depth ratio 
(a)

IEC-6 MTT Assay

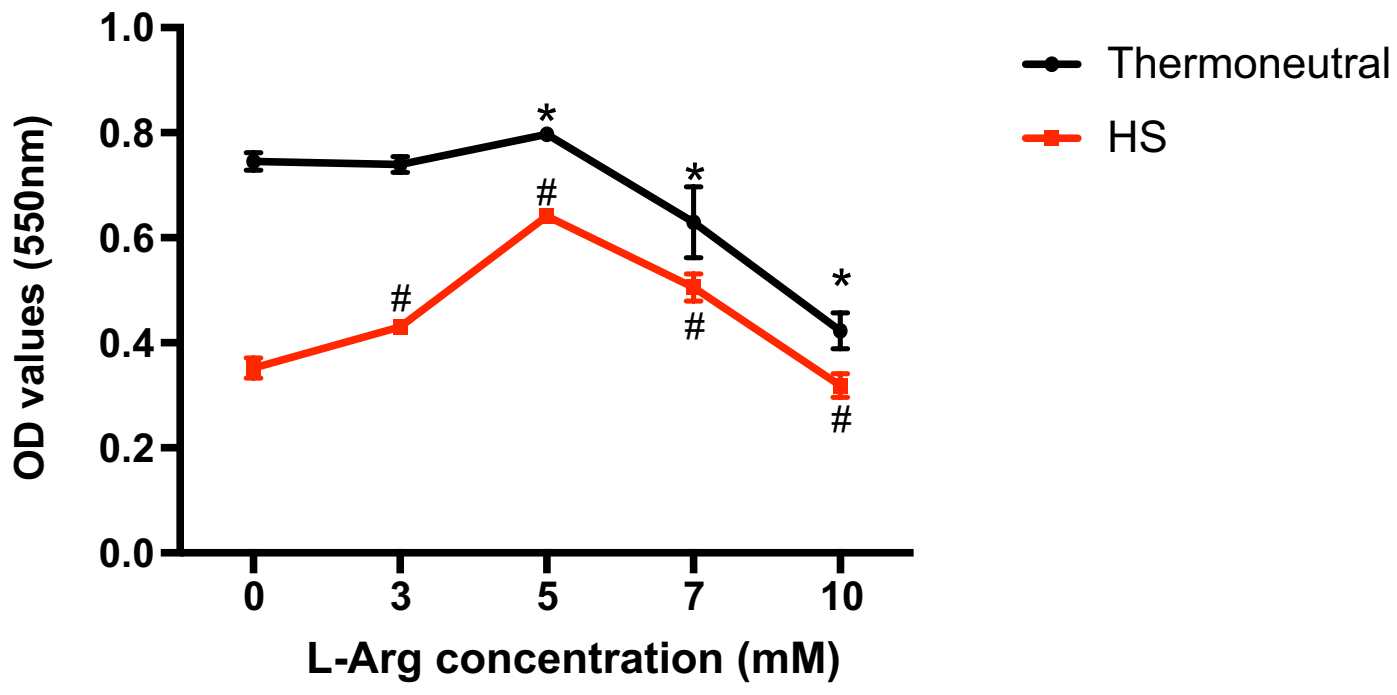

(b)
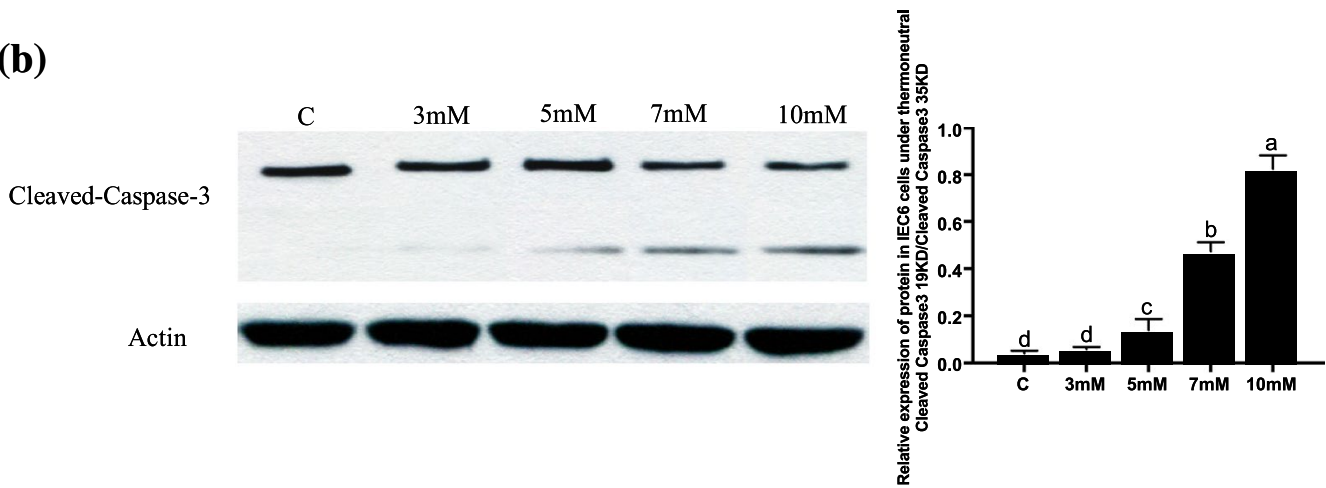

(c)

Actin
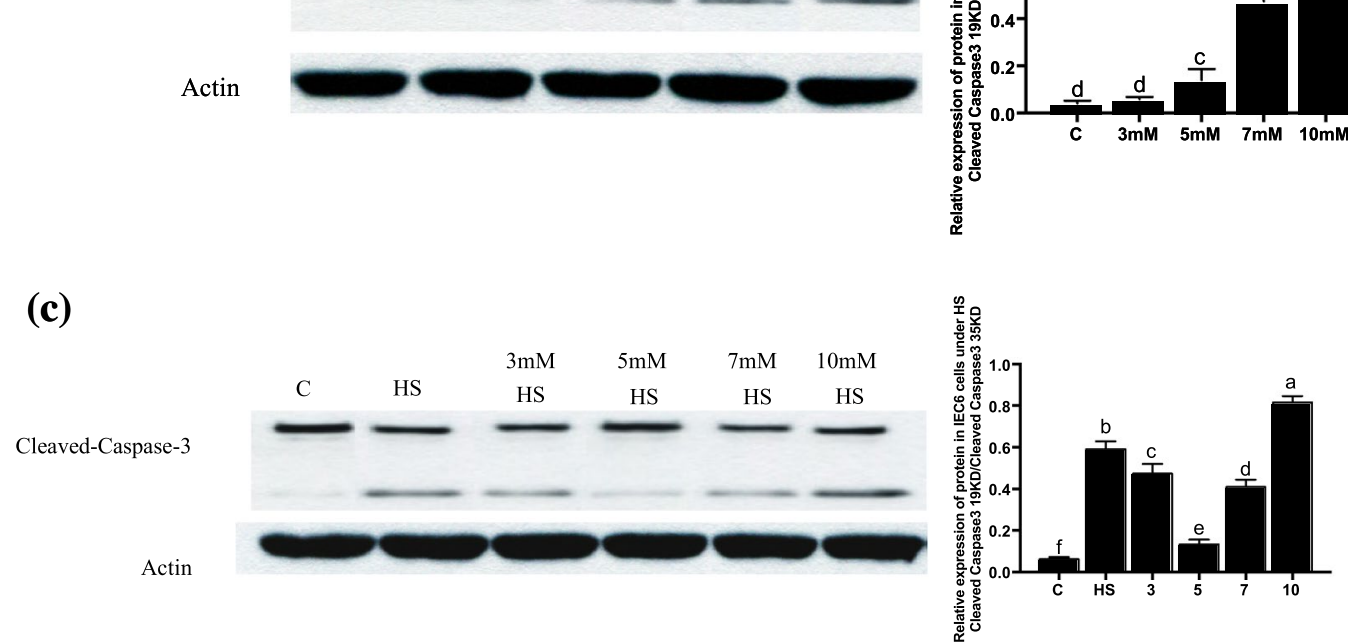

Fig. 1 a Effect of various concentrations of L-arginine on IEC-6 cell proliferation under thermoneutral and hyperthermic conditions determined by MTT assays. Expression of cleaved caspase- 3 protein in IEC- 6 cells under normal cultural condition (b) and subjected to heat exposure (c), as assessed by western blotting. Data are presented

was calculated. Epithelial cell apoptosis was detected by TdT-mediated dUTP nick-end labeling (TUNEL) with a TUNEL staining kit (Roche, Basel, Switzerland), according to the manufacturer's instructions. All observations were performed with a BH2 Olympus microscope 160 (DP71, Olympus, Tokyo, Japan) and ProgRes CapturePro software (version 2.7, Jenoptik, Jena, Germany). as the mean $\pm \mathrm{SD}, \mathrm{n}=3 .{ }^{\dagger} C$ control, $H S$ heat stress, $3 m M 3 \mathrm{mmol}$ L-arginine + heat stress, $5 \mathrm{mM} 5 \mathrm{mmol} \mathrm{L}$-arginine + heat stress, $7 \mathrm{mM}$ $7 \mathrm{mmol} \mathrm{L}$-arginine + heat stress, $10 \mathrm{mM} 10 \mathrm{mmol} \mathrm{L}$-arginine + heat stress. ${ }^{*} P<0.05$ compared with the control group. ${ }^{\#} P<0.05$ compared with the heat stress group

\section{Immunohistochemical staining}

The expression of cleaved caspase-3, ZO-1 and claudin1 protein were assessed by immunohistochemistry. Sections of jejunum ( $5 \mu \mathrm{m}$ thick) were quenched in $3 \%$ hydrogen peroxide, blocked in 5\% goat serum, and then incubated with primary antibodies (anti-cleaved caspase-3, Cell Signaling 
Fig. 2 Differential gene expression and KEGG analysis of IEC-6 cells. a Two log foldchange values of the indicated genes in control, heat stress, and L-arginine cell groups. The expression gradient is indicated by the legend. L-Arginine increased gene expression of tight junction and cell adhesion proteins. b Enrichment of differentially expressed genes in KEGG pathway analysis (a)

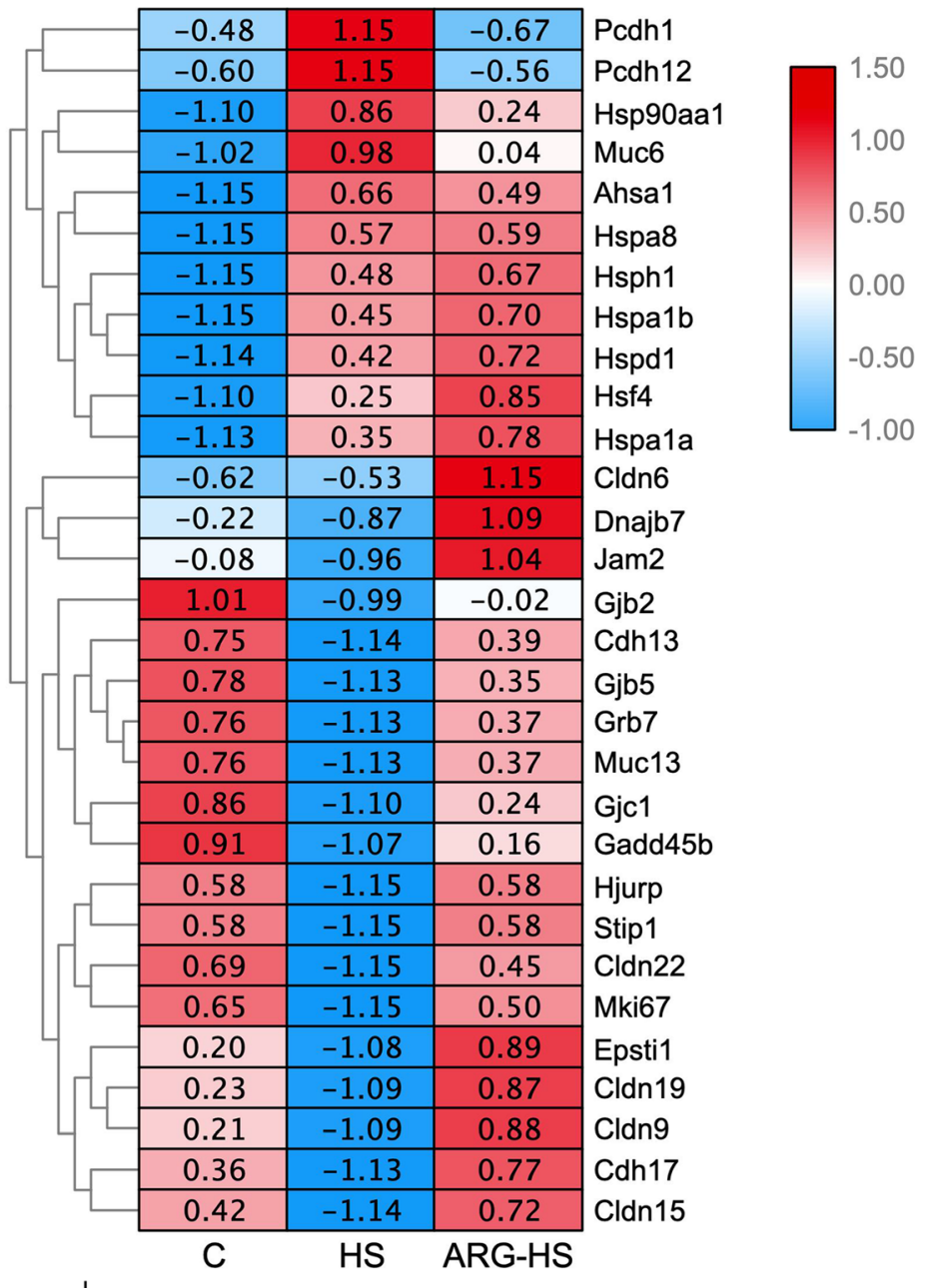

(b)

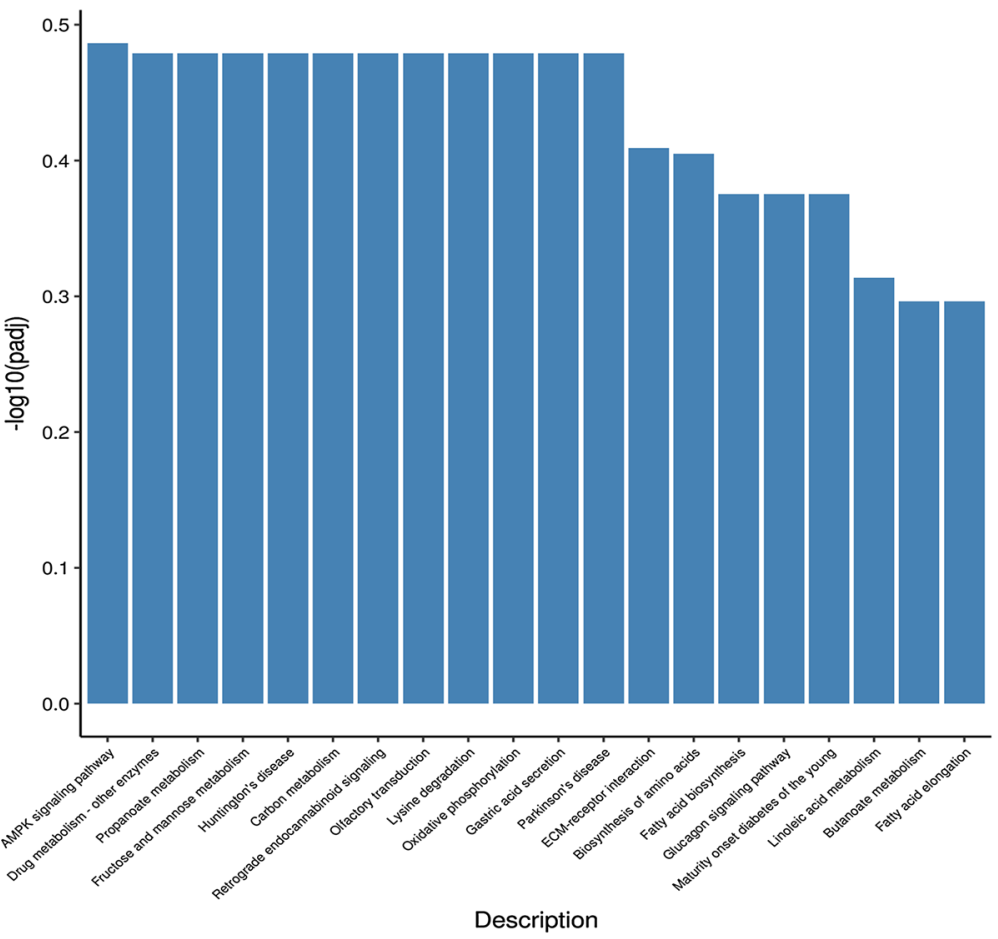


(a)

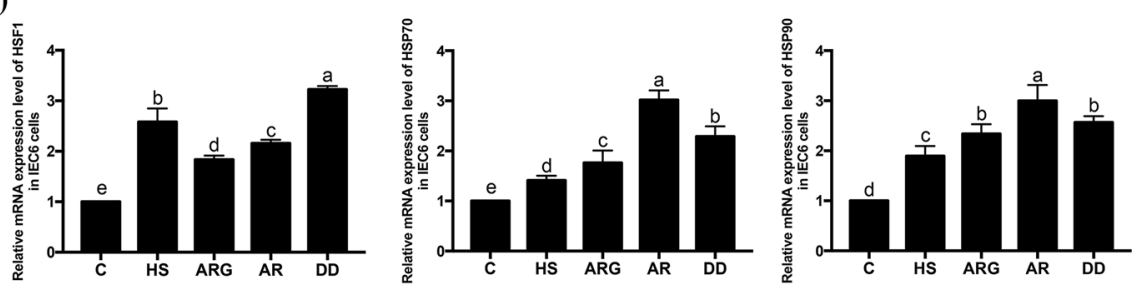

(b)

(c)
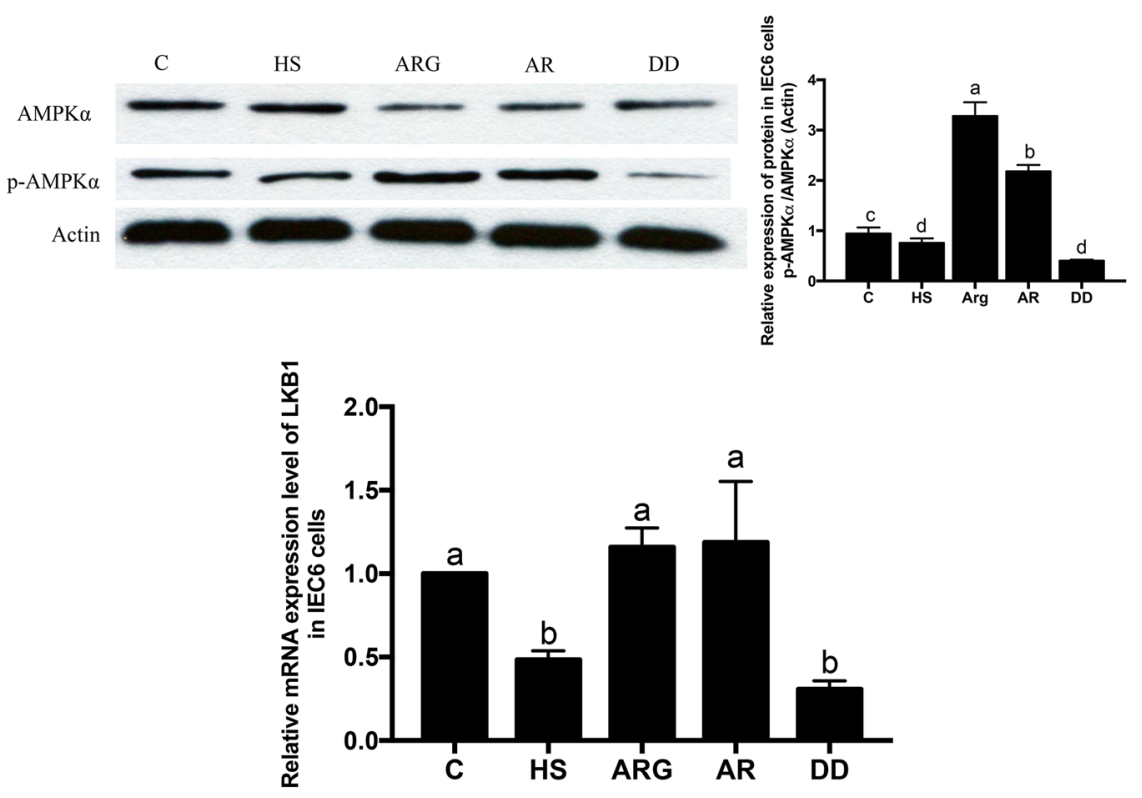

(d)
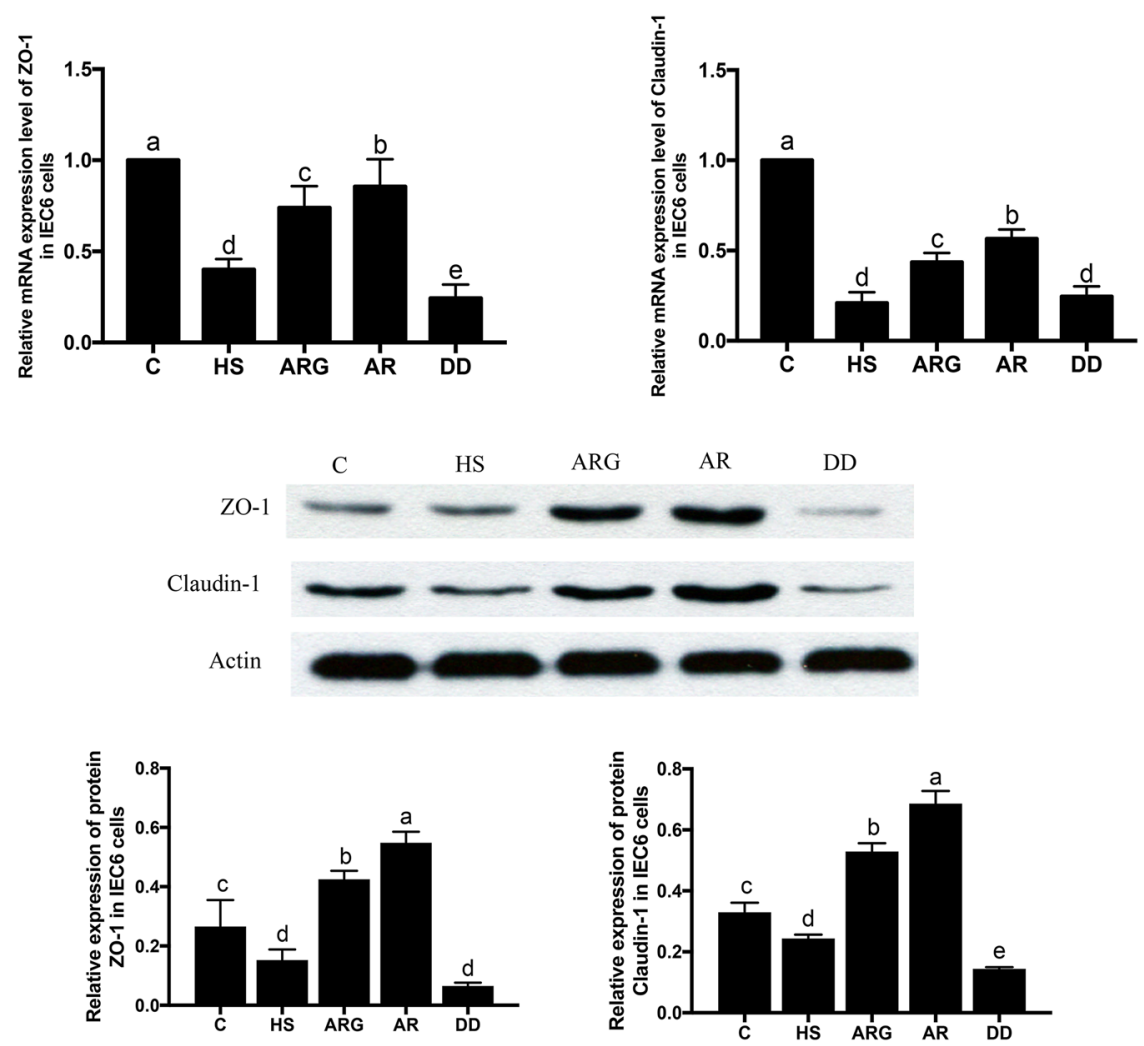
4Fig. 3 L-Arginine activates the AMPK signaling pathway under heat stress in IEC-6 cells. a Expression of heat shock protein-related genes in IEC-6 cells assessed by RT-qPCR. b Expression levels of $\mathrm{AMPK} \alpha$ and $\mathrm{p}-\mathrm{AMPK} \alpha$ proteins assessed by western blotting. c mRNA expression level of LKB1 assessed by RT-qPCR. d mRNA and protein expression levels of ZO-1 and claudin-1 assessed by RT- qPCR and western blotting, respectively. Data are presented as the mean $\pm \mathrm{SD}, \mathrm{n}=3 .{ }^{\dagger} C$ control, $H S$ heat stress, $L A$ L-arginine + heat stress, AR AICAR + heat stress, DD dorsomorphin dihydrochloride + heat stress. ${ }^{a-e}$ Bars with letters indicate significant difference among different treatments $(P<0.05)$

Technology; anti-ZO-1, Abcam; anti-claudin-1, Abcam) at $4{ }^{\circ} \mathrm{C}$ overnight. Subsequently, the sections were incubated with the secondary antibody for $30 \mathrm{~min}$. Immunoreactivity was detected by incubation in $0.1 \%$ 3,3-diaminobenzidine and $0.02 \%$ hydrogen peroxide for $5 \mathrm{~min}$ (Vector Laboratories, Burlingame, CA, USA).

\section{Total RNA extraction and RT-qPCR}

Total RNA of IEC- 6 cells and jejunum were extracted by TRIzol reagent (Invitrogen), according to the manufacturer's protocol. The RNA concentration and purity were measured by a nanodrop spectrophotometer (ND-2000, Thermo Fisher Scientific) based on the OD260/280 ratio. Total RNA $(1 \mu \mathrm{g})$ was used for reverse transcription with a commercial RNA transcription kit (CWbio Co., Ltd., Beijing, China) in a $20-\mu \mathrm{L}$ reaction including $4 \mu \mathrm{L}$ reverse transcriptase reaction buffer, $2 \mu \mathrm{L}$ DTT, $1 \mu \mathrm{L}$ dNTPs, $1 \mu \mathrm{L} \mathrm{HiFi-Moloney} \mathrm{murine}$ leukemia virus transcriptase, and RNase-free water, following the manufacturer's protocol. The cDNA was stored at $-20{ }^{\circ} \mathrm{C}$.

The expression levels of genes were determined by realtime quantitative PCR (RT-qPCR). Gene primer sequences are listed in Table 1. RT-qPCR was performed on a Line Gene 9600 Plus (Bioer Technology, Hangzhou, China) using a SYBR-Green kit (CWbio Co., Ltd.). The protocol was as follows: initial denaturation at $95{ }^{\circ} \mathrm{C}$ for $10 \mathrm{~min}$, followed by 45 cycles at $95^{\circ} \mathrm{C}$ for $15 \mathrm{~s}$ and $60{ }^{\circ} \mathrm{C}$ for $60 \mathrm{~s}$ for annealing and extension. The specificity of the amplified products was evaluated by melting curve analysis. $\beta$-Actin was used as an endogenous reference gene. The average gene expression level relative to $\beta$-actin mRNA in each sample was calculated using the $2^{-\Delta \Delta \mathrm{Ct}}$ method.

\section{Protein extraction and western blotting}

Jejunum tissue and IEC-6 cells were homogenized using lysis buffer containing phosphatase and protease inhibitors (Beyotime Institute of Biotechnology, Shanghai, China). The protein concentration was determined by a BCA protein assay kit (Beyotime Institute of Biotechnology). Proteins were separated by SDS-PAGE and transferred to polyvinylidene fluoride membranes (Sigma). The membranes were then incubated overnight at $4{ }^{\circ} \mathrm{C}$ with primary antibodies as follows: anti-cleaved caspase-3 (1:800, Cell Signaling Technology, Danvers, MA, USA), anti-AMPK $\alpha$ (1:1000, Sigma), anti-p-AMPK $\alpha$ (1:500, Cell Signaling Technology), anti-claudin-1 (1:500, Abcam, Cambridge, UK), antiZO-1 (1:500, Abcam), and anti-actin (1:3000, Abcam). After washing three times with TBST, the membranes were incubated with goat anti-mouse/rabbit IgG-HRP (1:5000) as the secondary antibody for $50 \mathrm{~min}$ at room temperature. The membranes were then exposed and to light-sensitive film. Quantification of western blot images was performed with Quantity One (v.4.6.2; Bio-Rad, Hercules, CA, USA).

\section{Statistical analysis}

All data were analyzed for statistical significance by one-way ANOVA using SPSS 17.0 software (version 17.0, SPSS Inc., Chicago, IL, USA). Data are presented as the mean \pm SD of at least three replicates for independent experiments. Differences were considered statistically significant at $P<0.05$. All the figures presented in this paper were created using GraphPad Prism (version 7.0, GraphPad Software, San Diego, USA).

\section{Results}

\section{Effect of L-arginine on cell proliferation and apoptosis}

As shown in Fig. 1a, at normal temperature, $5 \mathrm{mmol}$ L-arginine significantly promoted IEC-6 cell proliferation $(P<0.05)$. At higher $\mathrm{L}$-arginine concentrations, 7 and $10 \mathrm{mmol}$, cell proliferation was suppressed compared with the control group $(P<0.05)$. However, 3,5 , and 7 mmol L-arginine markedly increased cell proliferation after heat exposure $(P<0.05)$, and $5 \mathrm{mmol}$ had the optimal effect on IEC-6 cell proliferation. L-arginine at $10 \mathrm{mmol}$ worsened the damage of heat stress in cells and further suppressed cell proliferation $(P<0.05)$. Additionally, western blotting (Fig. 1b, c) showed the protein expression levels of cleaved caspase- 3 in different thermal conditions. The supplementation of L-arginine at 5, 7 and $10 \mathrm{mmol}$ increased the protein expression level of cleaved caspase-3 under thermoneutral condition, especially the 7 and 10 mmol treatment (Fig. 1b). On the other hand, heat stress significantly elevated the protein expression level of cleaved caspase-3, particularly under treatment with $10 \mathrm{mmol} \mathrm{L-arginine} \mathrm{(Fig.} \mathrm{1c),} \mathrm{indicat-}$ ing severe cell death. L-Arginine at 3, 5, and $7 \mathrm{mmol}$ attenuated protein expression of cleaved caspase-3, and $5 \mathrm{mmol}$ 
(a)
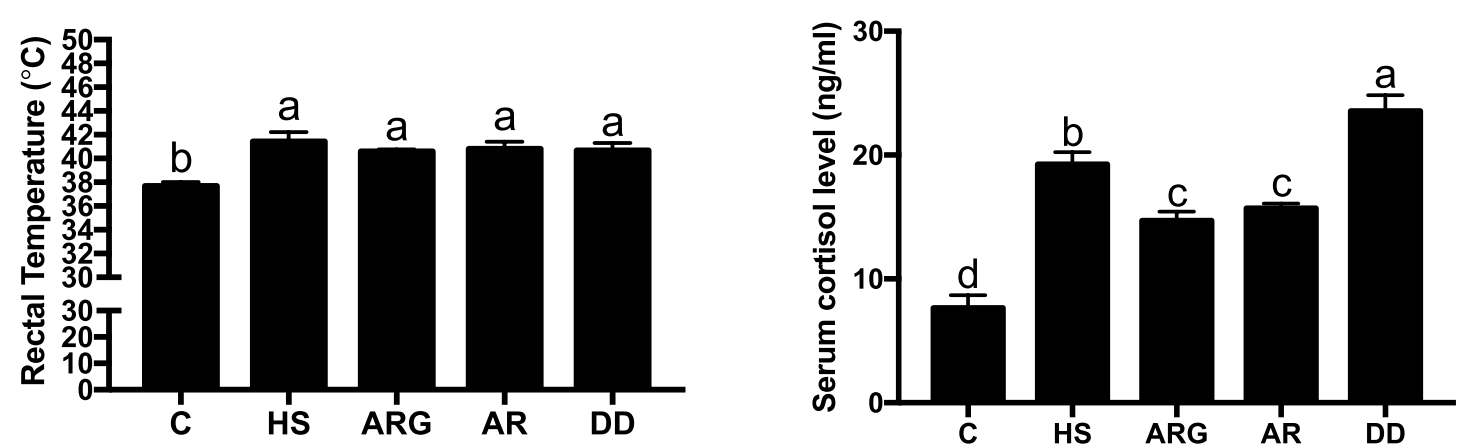

(b)
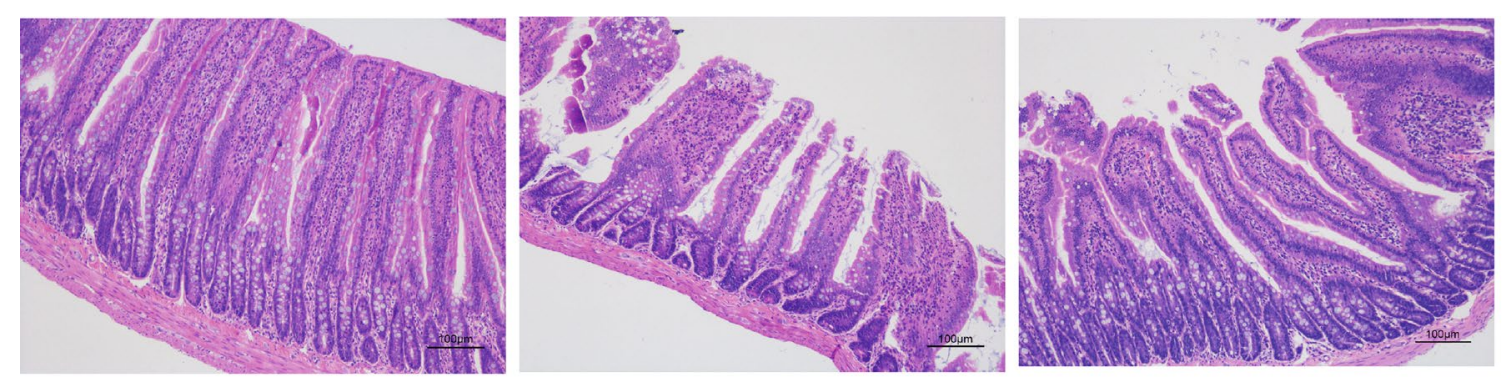

C

HS

ARG

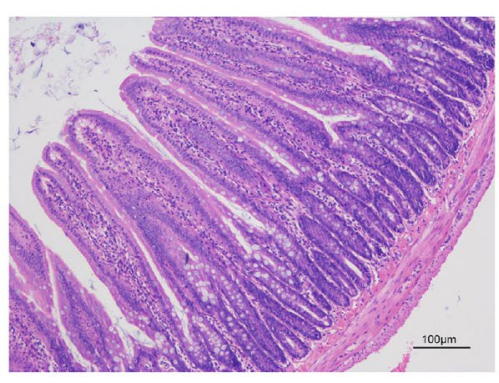

AR

(c)

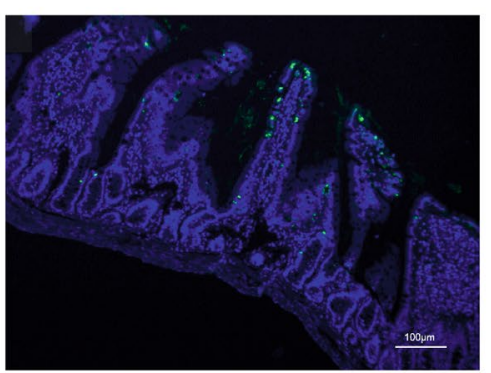

C

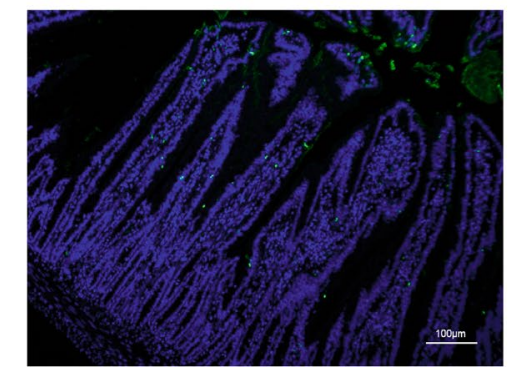

AR

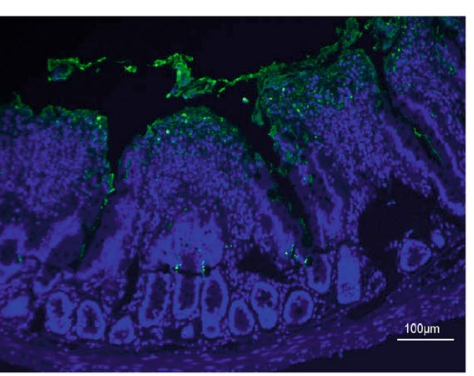

HS

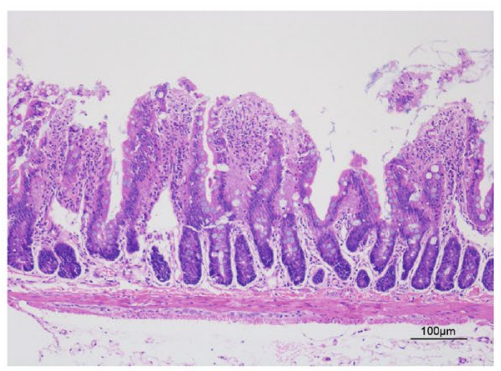

DD

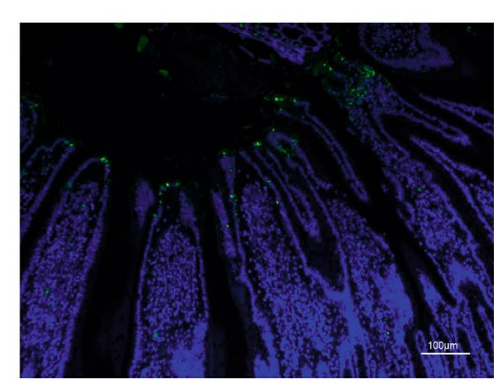

ARG 
4 Fig. 4 Effects of L-arginine on the stress response and intestine morphology. a Physiological changes of the various groups. L-Arginine and AICAR significantly decreased the serum level of cortisol. b Hematoxylin and eosin-stained sections of rat jejunum. Heat stress and dorsomorphin treatment shortened the villus height and caused swelling of jejunal villi. The intestinal structure was preserved by L-arginine and AICAR treatments. c TUNEL staining of the rat jejunum in the various groups. Cell nuclei were stained with DAPI. d
Rat jejunum stained with an antibody against caspase-3. e Changes in serum D-lactate and diamine oxidase of the various groups after heat stress. $\mathbf{f}$ Expression level of MLCK assessed by RT-qPCR. Data are presented as the mean $\pm \mathrm{SD}, \mathrm{n}=8 .{ }^{\dagger} C$ control, $H S$ heat stress, $L A$ L-arginine + heat stress, $A R$ AICAR + heat stress, $D D$ dorsomorphin dihydrochloride + heat stress. ${ }^{\mathrm{a}-\mathrm{e}} \mathrm{B}$ ars with letters indicate significant differences among the various treatments $(P<0.05)$

(d)

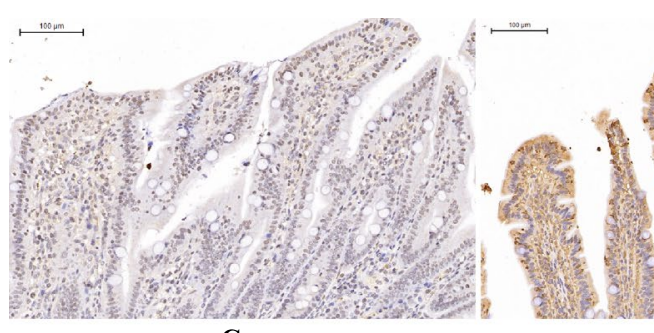

C
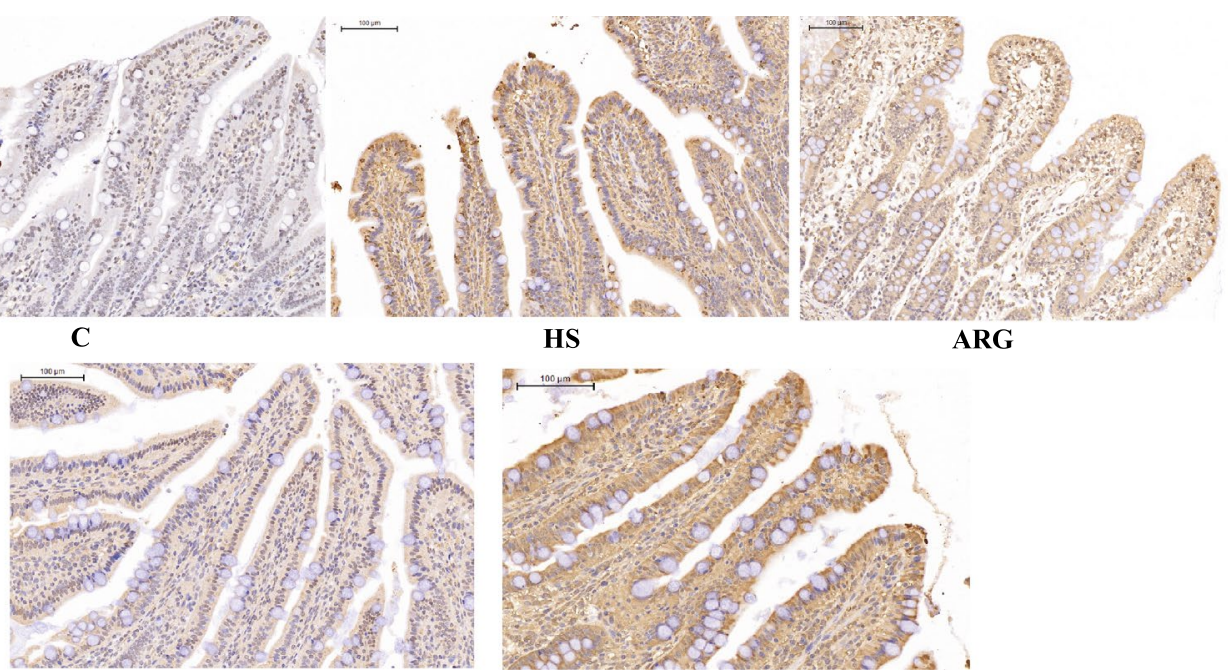

(e)

AR

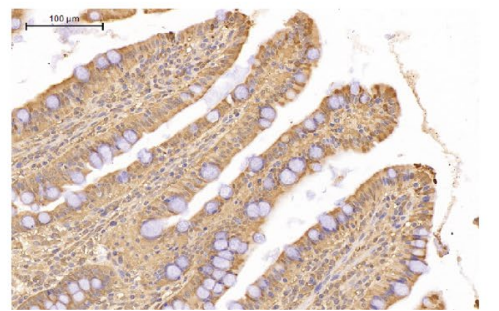

DD
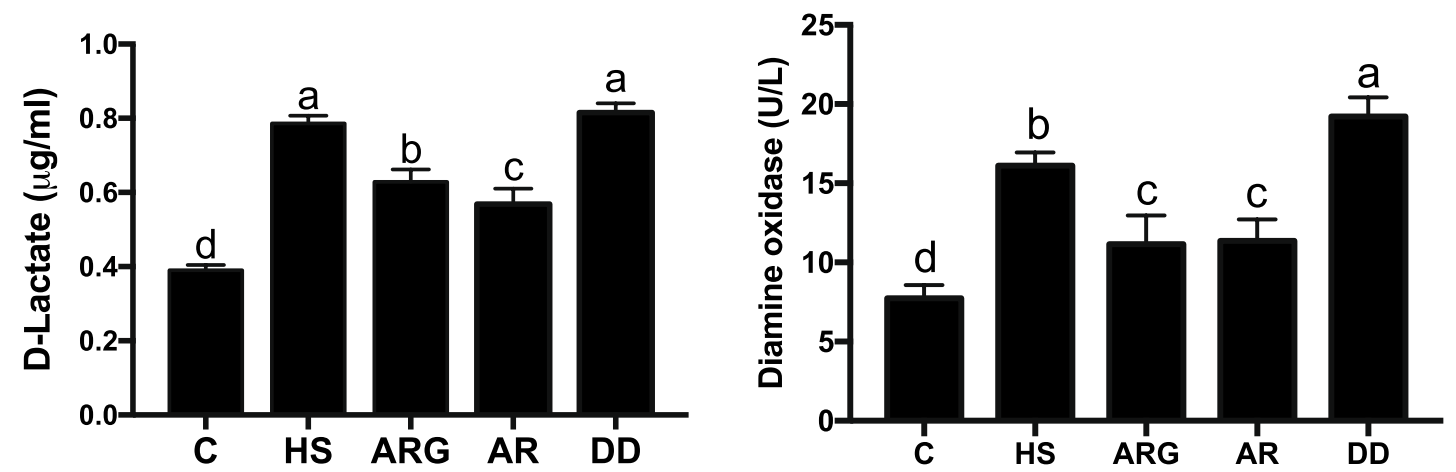

(f)

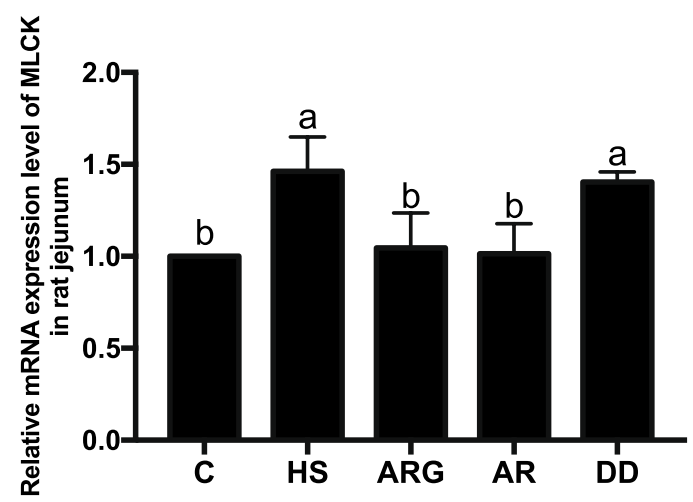

Fig. 4 (continued) 
L-arginine exerted the optimal effect under heat stress. According to these results, $5 \mathrm{mmol} \mathrm{L}$-arginine was considered to be the appropriate concentration for further study.

\section{Differentially expressed genes related to heat shock proteins and tight junction proteins, and KEGG pathway analysis}

The gene expression profile of IEC- 6 cells was obtained by RNA transcriptome sequencing of control, heat stress, and L-arginine groups. Figure 2a shows that heat stress enhanced the expression level of heat shock protein-related genes Hsp90aa1, Ahsa1, Hspa8, Hsph1, Hspa1b, Hspd1, Hsf4, Hspa1, and Dnajb7, and the log values were higher in the ARG group, indicating that $\mathrm{L}$-arginine promoted the expression of these genes to facilitate adjustment to hyperthermia. L-Arginine also enhanced cell proliferation and decreased apoptosis under heat stress as evidenced by increased expression of Gadd45b, Stip1, Mki67, and Grb7. Heat exposure increased expression of Pcdh1 and pcdh12, and decreased expression of tight junction proteins (Muc13, Cldn6, Cdh13, Cldn22, Cldn19, Cldn9, Cdh17, and Cldn15) and other proteins correlated with epithelial cell adhesion (Jam2, Gjb2, Gjb5, Gjc1, Hjurp, and Epstil1). However, the L-arginine group showed opposing results with increased gene expression of tight junction and adhesion proteins. These data suggested that L-arginine was beneficial to the intestinal barrier under heat stress and might reduce gap junctional permeability. Furthermore, KEGG pathway analysis was performed to compare the enrichment of differentially expressed genes between ARG and HS groups (Fig. 2b). The results suggested that L-arginine was mainly involved in mediation of the AMPK pathway under heat stress.

\section{L-Arginine promotes intestinal tight junctions by activating the AMPK pathway in IEC- 6 cells after heat exposure}

Expression of HSF1, HSP70, and HSP90 in IEC-6 cells was assessed by RT-qPCR. As shown in Fig. 3a, heat treatment upregulated expression of these genes in all groups except for the $\mathrm{C}$ group. L-Arginine and AICAR treatments significantly decreased the HSF1 expression level $(P<0.05)$ and markedly increased HSP70 and HSP90 expression levels $(P<0.05)$ compared with the HS group. Dorsomorphin treatment markedly increased HSF1 expression $(P<0.05)$. HSP70 and HSP90 expression in the DD group was lower than that in the AR group. Western blotting (Fig. 3b) confirmed that the AMPK $\alpha$ pathway was activated by L-arginine with a higher expression level of p-AMPK $\alpha$. AR and DD groups served as positive and negative controls, respectively. The results indicated that $\mathrm{L}$-arginine had the same property as AICAR to stimulate the AMPK pathway under heat stress. Figure $3 \mathrm{c}$ shows that heat stress and dorsomorphin treatments markedly downregulated relative expression levels of LKB $(P<0.05)$. The mRNA and protein expression levels of ZO-1 and claudin- 1 were markedly decreased by heat stress and dorsomorphin, whereas their expression levels were significantly elevated by L-arginine and AICAR as compared to the heat treatment (Fig. 3d).

\section{L-Arginine alleviates HS-induced injury in rat intestines}

As shown in Fig. 4a, heat exposure significantly elevated core body temperature $(P<0.05)$ and the serum cortisol level $(P<0.05)$ compared with the control group, demonstrating establishment of the heat stress model. However, ARG and AR groups showed a lower cortisol concentration than the HS group $(P<0.05)$, indicating that $\mathrm{L}$-arginine and AICAR suppressed the intensity of the body stress response. Figure $4 \mathrm{~b}$ shows that HS and dorsomorphin induced shedding and swelling of the villus tip. Additionally, Table 2 shows that the damage caused by HS and dorsomorphin significantly decreased the villus/crypt ratio $(P<0.05)$. However, L-arginine and AICAR treatments maintained a relatively intact intestinal structure and markedly increased the villus/crypt ratio $(P<0.05)$. TUNEL staining (Fig. $4 c)$ was strongly positive in HS and DD groups, revealing obvious epithelial cell apoptosis in the villus tip after heat stress and inhibition of the AMPK pathway worsening cell death. In contrast, L-arginine and AICAR limited apoptosis under heat stress as evidenced by less positive staining. Furthermore, we detected the expression level of caspase- 3 by immunohistochemistry and found that L-arginine and AICAR significantly reduced the expression of caspase-3 (Fig. 4d) after heat treatment. Moreover, HS and dorsomorphin significantly increased the serum D-lactate level, diamine oxidase activity, and expression of MLCK (Fig. 4e, f). However, L-arginine, which shared the same effect as AICAR, markedly decreased the serum D-lactate concentration, diamine oxidase activity, and relative mRNA expression level of MLCK (Fig. 4e, f).

\section{L-Arginine strengthens intestinal tight junctions by stimulating the AMPK pathway in the rat jejunum under heat stress}

Heat stress significantly increased the expression levels of HSF1, HSP70, and HSP90 in HS, ARG, AR and DD groups (Fig. 5a). Compared with the HS group, L-arginine and AICAR significantly downregulated the expression of HSF1 $(P<0.05)$ and upregulated the expression of HSP70 and HSP90 $(P<0.05)$. Dorsomorphin treatment 
Table 2 Effects of L-arginine on the jejunum morphology of rats

\begin{tabular}{lllllllll}
\hline Items & \multicolumn{2}{l}{ Treatments } & \multicolumn{3}{l}{ SD } & $P$-value \\
\cline { 2 - 6 } & C & HS & ARG & AR & DD & \\
\hline Villus height (mm) & $0.57 \pm 0.36^{\mathrm{a}}$ & $0.42 \pm 0.25^{\mathrm{c}}$ & $0.50 \pm 0.23^{\mathrm{b}}$ & $0.54 \pm 0.29^{\mathrm{ab}}$ & $0.38 \pm 0.25^{\mathrm{c}}$ & 0.768 & $<0.01$ \\
Crypt depth (mm) & $0.16 \pm 0.13^{\mathrm{b}}$ & $0.22 \pm 0.31^{\mathrm{a}}$ & $0.18 \pm 0.23^{\mathrm{b}}$ & $0.18 \pm 0.16^{\mathrm{b}}$ & $0.23 \pm 0.32^{\mathrm{a}}$ & 0.360 & $<0.01$ \\
Villus/crypt ratio & $3.63 \pm 0.22^{\mathrm{a}}$ & $1.92 \pm 0.27^{\mathrm{c}}$ & $2.82 \pm 0.32^{\mathrm{b}}$ & $3.04 \pm 0.31^{\mathrm{b}}$ & $1.68 \pm 0.23^{\mathrm{c}}$ & 0.780 & $<0.01$ \\
\hline
\end{tabular}

$C$ control, $H S$ heat stress, $L A$ L-arginine + heat stress, $A R$ AICAR + heat stress, $D D$ dorsomorphin dihydrochloride + heat stress

${ }^{\mathrm{a}-\mathrm{e}}$ Means in the same row, values with different small letter superscripts show significant difference $(P<0.05)$ markedly increased HSF1, HSP70, and HSP90 expression levels $(P<0.05)$ compared with the HS group. Additionally, western blotting demonstrated that the models of activating (AICAR) and inhibiting (dorsomorphin) the AMPK pathway in rat intestines were established (Fig. 5b) with a dramatic elevation and decrease in the expression of $\mathrm{p}-\mathrm{AMPK} \alpha$, respectively. L-arginine activated the AMPK pathway by significantly increasing the expression $\mathrm{p}-\mathrm{AMPK} \alpha$. Furthermore, relative mRNA expression of LKB1 was dramatically decreased $(P<0.05)$ under heat stress and by dorsomorphin treatment (Fig. 5c). As shown in Fig. 5d, hyperthermia and the AMPK inhibitor markedly suppressed both the mRNA and protein expression levels of $\mathrm{ZO}-1$ and claudin- 1 compared to the control group. However, the L-arginine and the AMPK activator significantly enhanced the expression of these tight junction-related genes and proteins compared to the heat stroke treatment. Additionally, protein expression levels of ZO-1 and claudin-1 were also detected by immunohistochemistry, L-arginine and AICAR significantly increased the expression of both ZO-1 and claudin-1, whereas heat stress and dorsomorphin markedly reduced their expression (Fig. 5e, f).

\section{Discussion}

In the present study involving both an intestinal epithelial cell line (IEC-6) and rat intestine model, we showed that L-arginine is critical for intestinal epithelial barrier integrity, particularly under heat stress conditions, because it enhances the expression and production of TJ proteins by regulating the AMPK signaling pathway.

L-Arginine has multiple beneficial effects on the intestines $[19,26]$, although we found that its protective effect was only exerted in a specific concentration range. As shown in Fig. 1a, L-arginine concentrations exceeding $5 \mathrm{mmol}$ suppressed cell proliferation and was even cytotoxic in the optimized culture environment. Heat stress increases the requirement of nutrition for cells to survive [29]. However, the protective effect was not lineally dependent on dose, and a high dose of L-arginine $(10 \mathrm{mmol})$ decreased cell viability.
The effect of various concentrations of L-arginine on IEC-6 cells apoptosis under normal condition and heat stress treatment were also analyzed by western blotting (Fig. 1b, c). L-arginine at $5 \mathrm{mmol}$ minimized the expression level of cleaved caspase- 3 after $1.5 \mathrm{~h}$ of heat exposure. Hence, $5 \mathrm{mmol}$ was chosen as the optimal concentration for further study.

To further investigate how L-arginine was involved in the regulation of gene expression and signaling pathways under heat stress, we analyzed the transcriptome in IEC-6 cells of the various groups. Some studies have demonstrated that heat stress leads to the elevation of HSPs [11-13]. This study obtained similar results with higher expression levels of HSPs in HS and ARG groups (Fig. 2a). L-Arginine also significantly upregulated genes of the tight junction protein family and adhesion junction proteins, indicating that L-arginine strengthened the intercellular junctions between epithelial cells, which were disrupted by hyperthermia. Moreover, KEGG pathway analysis of HS and ARG groups revealed that the AMPK pathway was activated by L-arginine (Fig. 2b).

Heat stress is an important factor influencing domestic animal performance and causing gastrointestinal tract dysfunction during summer $[1,4]$. HSPs are widely known to be involved in various physiological processes and play a key role in the stress response by limiting the damage caused by stress and promoting cell recovery [30]. Hao et al. [12] and Gao et al. [11] found that HSP70 and HSP90 increase in rat intestines after exposure to heat. Similarly, the current study revealed marked elevation of HSP70 and HSP90 in both cells and rat intestines after heat exposure. Heat shock factors (HSFs), biomarkers of stress-induced tissue injury, undergo phosphorylation in response to stress and enhance the transcription of HSPs [8]. Consistently, we found that HS dramatically increased the expression level of HSF1 both in vitro and in vivo. Rectal temperature and serum cortisol are commonly considered as indicators of heat stress and were higher in the four heat treatment groups compared with the control group. The alterations of these parameters, together with the increased expression levels of HSF1, HSP70, and HSP90, confirmed hyperthermia. L-Arginine as 
Fig. 5 L-Arginine activates the AMPK pathway after heat stress in rat intestines. a Expression of heat shock protein-related genes in intestines measured by RT-qPCR. b Expression levels of AMPK $\alpha$ and p-AMPK $\alpha$ proteins assessed by western blotting. L-Arginine increased the expression level of $\mathrm{p}-\mathrm{AMPK} \alpha$ in intestines. c mRNA expression level of LKB1 assessed by RT-qPCR. $\mathbf{d}$ mRNA and protein expression levels of ZO- 1 and claudin- 1 assessed by RT-qPCR and western blotting, respectively. e The expression level of ZO-1 protein were evaluated in jejunal specimens by immunohistochemistry. f The expression of claudin-1 protein in rats jejunum tissues. Data are presented as the mean $\pm \mathrm{SD}$, $\mathrm{n}=8 .{ }^{\dagger} C$ control, $H S$ heat stress, $L A$ L-arginine + heat stress, $A R$ AICAR + heat stress, $D D$ dorsomorphin dihydrochloride + heat stress. ${ }^{\mathrm{a}-\mathrm{e}}$ Bars with letters indicate significant differences among various treatments $(P<0.05)$ (a)
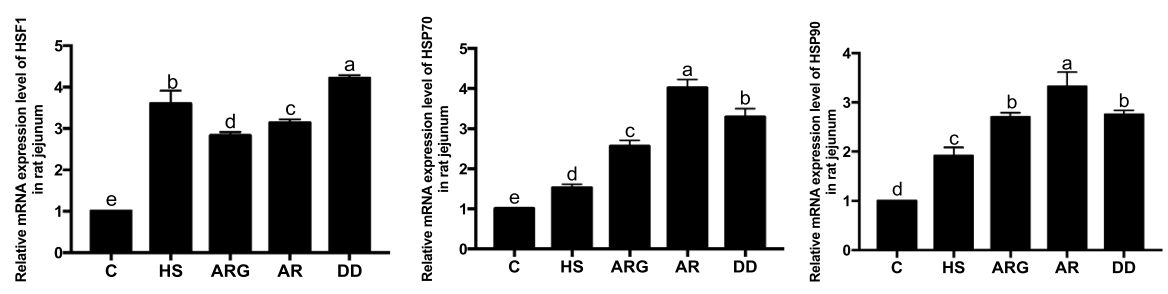

(b)
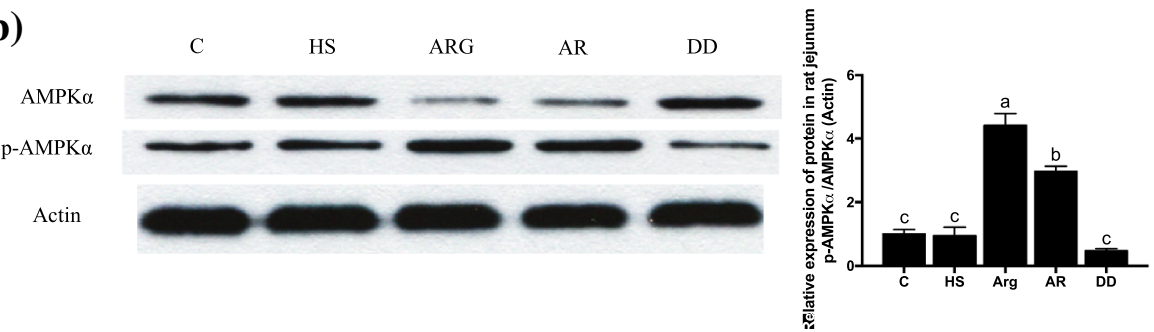

(c)

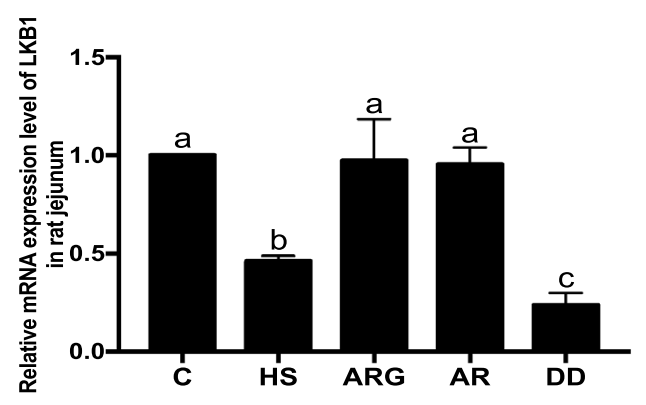

(d)

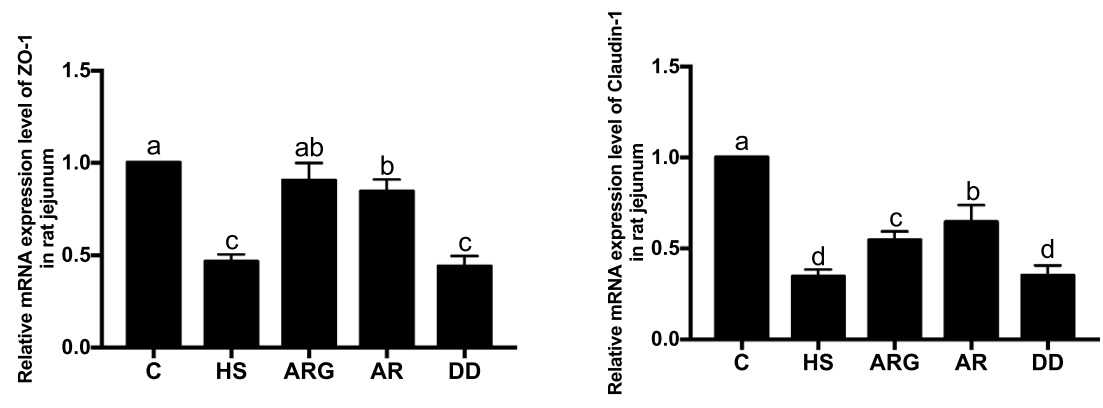

C

HS ARG

AR

DD

ZO-1

Claudin-1

Actin
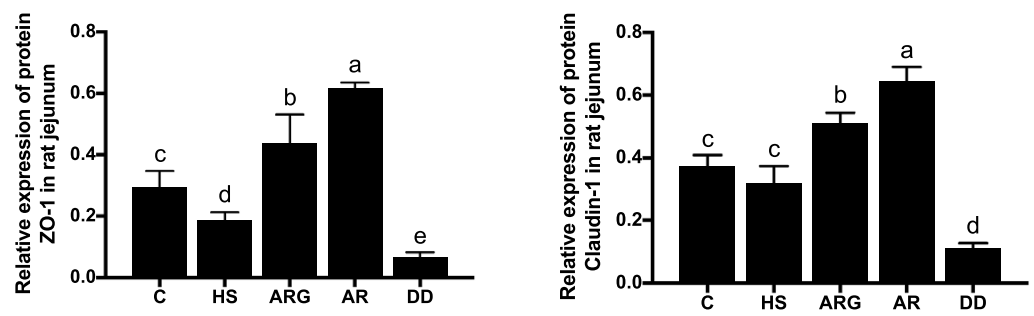
(e)

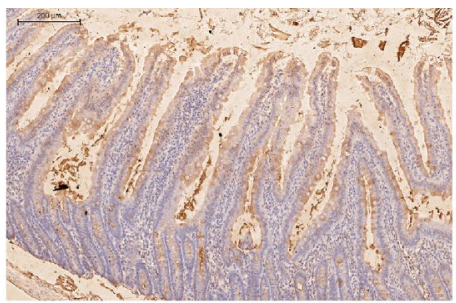

C

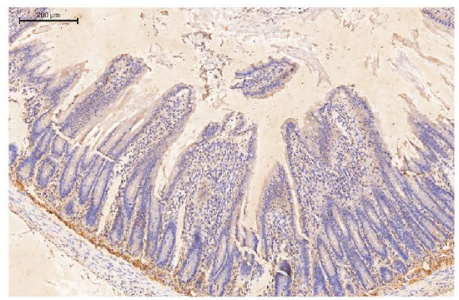

HS

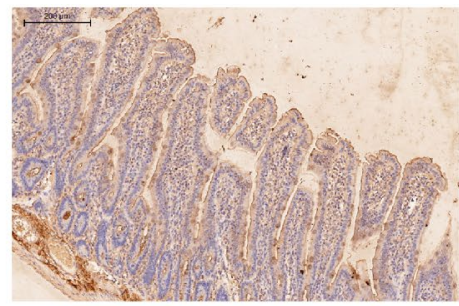

ARG
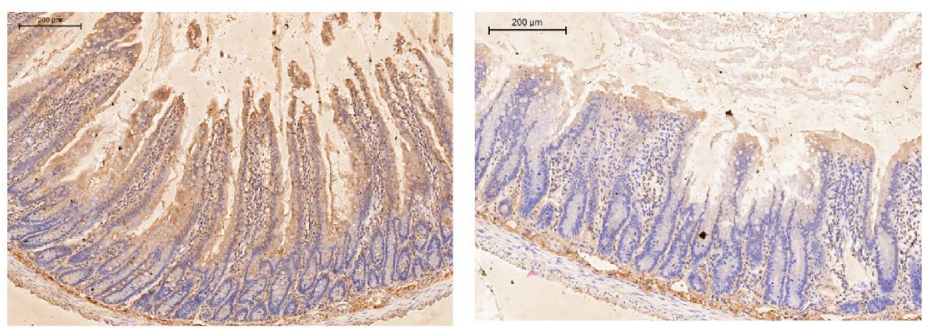

(f)

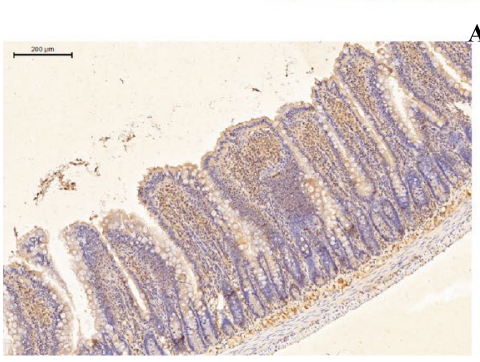

C
AR

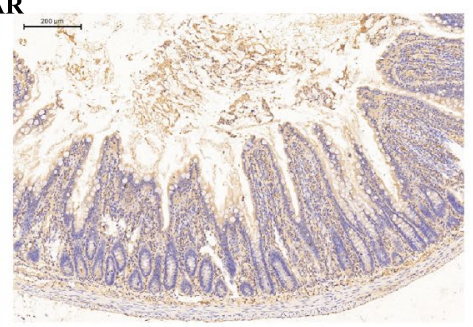

DD

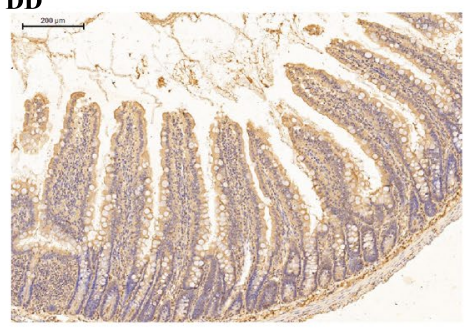

ARG

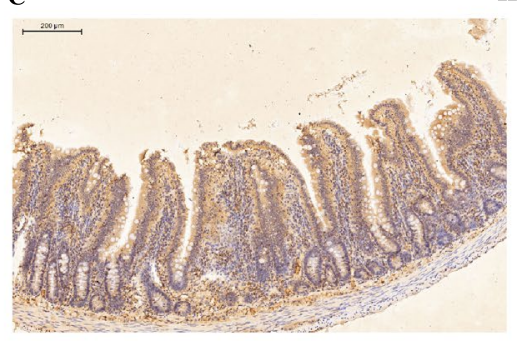

AR
HS

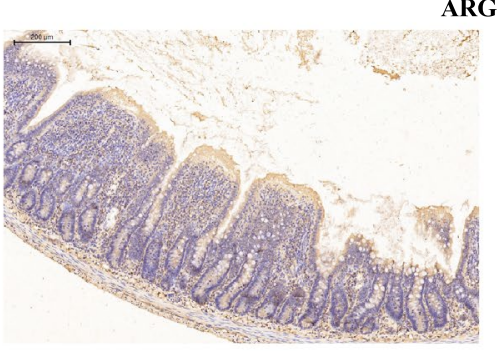

DD

Fig. 5 (continued)

a semi-essential amino acid facilitates maintenance of internal homeostasis [31]. The present study showed that, compared with the HS group, L-arginine significantly decreased HSF1 expression and increased HSP expression to assist IEC-6 cells and rats to adapt to heat. Moreover, it attenuated the body stress response by reducing the cortisol level during heat exposure.

The small intestine serves as a barrier to pathogens and provides the largest area for nutrient absorption. However, these functions can easily be compromised by heat shock [32]. Blood redistribution reduces blood supplying the small intestine to preserve the blood flow of vital organs such as the heart and brain. Several studies have demonstrated that hyperthermia causes intestinal damage and disrupts the intestinal structure $[33,34]$. In the current study, we also found that heat treatment induced intestinal injuries in rats, including a shortened villus height, epithelial cell shedding, and villus swelling. Morphological injuries were also observed by TUNEL staining and caspase-3 immunohistochemical analysis, indicating that heat stress caused extensive apoptosis in tissue, especially in the villus tip from the jejunum. Previous studies have demonstrated that $\mathrm{L}$-arginine improves mucosal development and recovery following intestinal injury in rats [26,27]. Similarly, the present study showed that L-arginine increased the villus height and villus/ 
crypts ratio after exposure to heat, thereby maintaining intestinal integrity.

Previous studies have shown that heat stress disrupts intestinal permeability [12, 35]. Yang et al. [35] found that the activity of MLCK, a protein kinase, is triggered by hyperthermia, which enhances the contractility of acto-myosin ring widening of the intracellular space. Consistently, our study showed that the actin cytoskeleton was damaged following heat stress with an increase in MLCK gene expression. Furthermore, intestinal permeability can be evaluated by blood indicators. Serum D-lactate and DAO are considered as biomarkers for barrier permeability [2,36]. D-Lactic acid is produced by intestinal flora and released into circulation following intestinal mucosa damage. Diamine oxidase is secreted by the epithelium, and its blood concentration increases upon intestine injury [37]. We obtained similar results in this study. Specifically, after 3 consecutive days of HS, the D-LAC level and DAO activity were increased significantly. These results demonstrated that the intestinal barrier function was compromised. In contrast, L-arginine markedly downregulated the expression level of MLCK and decreased the serum concentrations of D-LAC and DAO. These results strongly indicated that L-arginine restored intestinal permeability and attenuated heat stress-induced tissue injury.

AMP-activated protein kinase (AMPK), a highly conserved serine/threonine kinase, is widely known as a regulator of energy homeostasis to promote ATP generation and energy restoration [21]. When the overall energy level in a cell deceases because of increased demands by various stimulates, AMPK is activated through phosphorylation by upstream kinases or directly triggered by an elevated AMP/ ATP ratio [38]. Under heat stress, AMPK phosphorylation is downregulated in HeLa and HEK293 cells [39]. Wang et al. [39] postulated that dephosphorylation of the AMPK $\alpha$ subunit inhibits AMPK activity in various cultured epithelial cell lines (293T, C2C12, HepG2, bEnd.3, Hepa1-6, and MIN6 cells). The phosphorylated AMPK $\alpha$ protein levels in the cultured IEC- 6 cell line and rat jejunum were decreased after thermal stress exposure (Figs. 3b, 5b), which is consist with the above study.

Luminal nutrients contribute to alterations in the morphology and functions of IECs and intestines [29]. L-Arginine stimulates cell proliferation [40], enhances the intestinal mucosal immune function [19], and improves intestinal recovery [26]. However, how L-arginine is involved in the regulation of TJ proteins under heat stress and the underlying mechanism is still unclear. To elucidate whether L-arginine activates the AMPK pathway to exert its protective effect against hyperthermia, we used AICAR and dorsomorphin as positive and negative controls, respectively, in the present study. AICAR is an AMPK activator that can be converted into an AMP analog, and dorsomorphin is a potent and selective AMPK inhibitor that competes with ATP.

Dorsomorphin induced a significant increase in HSF-1 after heat stress, reflecting the oxidative stress response in IEC- 6 cells and the intestines. This result is in line with a study by Uerlings et al. [13]. L-Arginine, which had the same effect as AICAR, markedly elevated the expression levels of HSP70 and HSP90 (Figs. 3a, 5a). These findings suggest that the AMPK signaling pathway participates in mediation of the heat-induced response, which also supports the results of differential gene expression and KEGG analysis (Fig. 2a, b).

Nalli et al. [41] reported that AMPK is a negative regulator of MLCK and is responsible for the contractility of intestinal muscles. Uerlings et al. [13] also found that AMPK inhibition causes an increase in MLCK gene expression. Therefore, it is conceivable that the MLCK expression level was significantly increased in rat intestines by dorsomorphin treatment following 3 days of heat stress. In contrast, L-arginine and AICAR markedly decreased its expression. These results imply that L-arginine suppresses intestinal smooth muscle hypercontractility caused by thermal stimulation by regulating the AMPK pathway.

The phosphorylates of liver kinase B (LKB1), one of the AMPK upstream kinases, activates AMPK following an increase in cellular AMP levels [22]. Activation of AMPK is abolished in cells lacking expression of LKB1 [21]. In the current study, we found that LKB1 was significantly reduced under heat shock in both IEC- 6 cells and intestines, which was the same effect as AMPK inhibition. Interestingly, L-arginine and AICAR treatments had no significant effect on the mRNA expression of LKB1 under elevated temperature. This result indicated that triggering AMPK pathway under heat stress may not be attributed to the activation of LKB1.

Accumulating studies have shown that activation of AMPK by pharmacological or nutraceutical approaches strengthens the epithelial barrier function [21, 24, 38]. AICAR treatment enhances the barrier function in Caco-2 cells [42]. Consistent with a previous report, AICAR dramatically decreased the D-lactate concentration and DAO activity in vivo following heat stress. Moreover, TUNEL and caspase-3 IHC staining showed that AICAR limited epithelial cell apoptosis due to thermal injury. In contrast, AMPK inhibition caused by disease or detrimental stimulators is concurrent with an impaired barrier function [43]. Recent studies have also revealed that such inhibition exaggerates DSS-induced colitis [42]. Similarly, in the present study, we found that dorsomorphin exacerbated HSinduced intestinal damage with higher D-lactate and DAO levels, and severe apoptosis. We also found that L-arginine exerted a similar effect on intestinal barrier functions as AICAR. 
AMPK is a key factor in the regulation of tight junctions $[21,24,42]$. These effects have been implicated in some previous studies indicating that AMPK promotes ZO-1 assembly in MDCK cells [44]. In addition, activation of AMPK by AICAR enhances ZO-1 relocation in Caco-2 cells [25, 42]. Consistently, after heat exposure, protein expression levels of ZO- 1 and claudin- 1 in AICAR and L-arginine groups were significantly upregulated both in vitro and in vivo. In addition, we found decreased ZO-1 and claudin-1 gene expression in cells and intestines subjected to heat stress or treated with dorsomorphin, which is line with a study by Peng et al. [25]. These results also confirmed the differentially expressed genes and KEGG pathways of the transcriptome analysis.

\section{Conclusions}

L-Arginine has been demonstrated to have various beneficial effects on intestine development and recovery under harmful stimuli. In this study, we found that L-arginine improved the mucosal integrity disrupted by heat stress by promoting expression of tight junctions in an AMPK-dependent manner. Moreover, L-arginine might activate the AMPK pathway by regulating other upstream kinases rather than LKB1. Further studies are needed to confirm which of the AMKP upstream kinase such as CaMKK2, is the target of L-arginine actions. Collectively our findings revealed the underlying mechanism responsible for the beneficial effect of $\mathrm{L}$-arginine on the intestinal barrier function.

Acknowledgements We thank Peng Yin and Fenghua Liu for their efforts in the experimental design and data analysis.

Author contributions LH designed this study, analyzed data, and drafted the manuscript. YL, JL, and CL assisted with animal experiments, sampling, and measurements. $\mathrm{WZ}$ and $\mathrm{ZZ}$ performed in vitro experiments, PY and FL participated in the study design and data analysis. FZ supervised coordination and the study design. All authors approved the final manuscript.

Funding This study was supported by the National Science Foundation of China (No. 31572584).

Data availability Data sharing is not applicable to this article as no datasets were generated or analyzed during the current study.

\section{Compliance with ethical standards}

Conflict of interests The authors declare that they have no competing interests.

Ethical approval The experimental protocols of this study were approved by the Animal Care and Use Committee of China Agricultural University (Permit Number: 20181109-6).
Open Access This article is distributed under the terms of the Creative Commons Attribution 4.0 International License (http://creativeco mmons.org/licenses/by/4.0/), which permits unrestricted use, distribution, and reproduction in any medium, provided you give appropriate credit to the original author(s) and the source, provide a link to the Creative Commons license, and indicate if changes were made.

\section{References}

1. St-Pierre NR, Cobanov B, Schnitkey G (2003) Economic losses from heat stress by US livestock industries. J Dairy Sci 86:E52-E77

2. Cui Y, Gu X (2015) Proteomic changes of the porcine small intestine in response to chronic heat stress. J Mol Endocrinol 55(3):277-293

3. Liu F, Yin J, Du M, Yan P, Xu J, Zhu X et al (2009) Heat-stressinduced damage to porcine small intestinal epithelium associated with downregulation of epithelial growth factor signaling. J Anim Sci 87(6):1941-1949

4. Lambert GP (2009) Stress-induced gastrointestinal barrier dysfunction and its inflammatory effects. J Anim Sci 87(Suppl 14):E101-E108

5. Varasteh S, Braber S, Garssen J, Fink-Gremmels J (2015) Galactooligosaccharides exert a protective effect against heat stress in a Caco-2 cell model. J Func Foods 16:265-277

6. Yavas C, Yavas G, Celik E, Buyukyoruk A, Buyukyoruk C, Yuce D et al (2018) Beta-hydroxy-beta-methyl-butyrate, L-glutamine, and L-arginine supplementation improves radiation-induce acute intestinal toxicity. J Diet Suppl. 3:1-16

7. Al-Zghoul MB, El-Bahr SM (2019) Basal and dynamics mRNA expression of muscular HSP108, HSP90, HSF-1 and HSF-2 in thermally manipulated broilers during embryogenesis. BMC Vet Res 15(1):83

8. Gupta SC, Sharma A, Mishra M, Mishra RK, Chowdhuri DK (2010) Heat shock proteins in toxicology: how close and how far? Life Sci 86(11):377-384

9. Kalmar B, Greensmith L (2009) Induction of heat shock proteins for protection against oxidative stress. Adv Drug Deliv Rev 61(4):310-318

10. Tang YC, Chang HC, Hayer-Hartl M, Hartl FU (2007) SnapShot: molecular chaperones, part II. Cell 128(2):412

11. Gao Z, Liu F, Yin P, Wan C, He S, Liu X et al (2013) Inhibition of heat-induced apoptosis in rat small intestine and IEC- 6 cells through the AKT signaling pathway. BMC Vet Res 9:241

12. Hao Y, Feng Y, Li J, Gu X (2018) Role of MAPKs in HSP70's protection against heat stress-induced injury in rat small intestine. Biomed Res Int 2018:1571406. https://doi. org/10.1155/2018/1571406

13. Uerlings J, Song ZG, Hu XY, Wang SK, Lin H, Buyse J et al (2018) Heat exposure affects jejunal tight junction remodeling independently of adenosine monophosphate-activated protein kinase in 9-day-old broiler chicks. Poult Sci 97(10):3681-3690

14. Harhaj NS, Antonetti DA (2004) Regulation of tight junctions and loss of barrier function in pathophysiology. Int J Biochem Cell Biol 36(7):1206-1237

15. Suzuki T (2013) Regulation of intestinal epithelial permeability by tight junctions. Cell Mol Life Sci 70(4):631-659

16. Hartsock A, Nelson WJ (2008) Adherens and tight junctions: structure, function and connections to the actin cytoskeleton. Biochim Biophys Acta 1778(3):660-669

17. Zeissig S, Bürgel N, Günzel D, Richter J, Mankertz J, Wahnschaffe U et al (2007) Changes in expression and distribution of claudin 2, 5 and 8 lead to discontinuous tight junctions and barrier dysfunction in active Crohn's disease. Gut 56(1):61-72 
18. Vetrano S, Rescigno M, Cera MR, Correale C, Rumio C, Doni A et al (2008) Unique role of junctional adhesion molecule-A in maintaining mucosal homeostasis in inflammatory bowel disease. Gastroenterology 135(1):173-184

19. Balmant BD, Araújo EON, Yabuki D, Novais AB, Genaro SC, Laposy CB et al (2018) Effects of L-arginine supplementation on leukogram, inflammatory bowel infiltrates and immunoglobulins with 5-FU use in rats. Nutr Cancer 70(2):249-256

20. Zhang C, Zhao XH, Yang L, Chen XY, Jiang RS, Jin SH et al (2017) Resveratrol alleviates heat stress-induced impairment of intestinal morphology, microflora, and barrier integrity in broilers. Poult Sci 96(12):4325-4332

21. Rowart P, Wu J, Caplan MJ, Jouret F (2018) Implications of AMPK in the formation of epithelial tight junctions. Int $\mathrm{J}$ Mol Sci 19(7):E2040. https://doi.org/10.3390/ijms 19072040

22. Hawley SA, Boudeau J, Reid JL, Mustard KJ, Udd L, Mäkelä TP et al (2003) Complexes between the LKB1 tumor suppressor, STRAD alpha/beta and MO25 alpha/beta are upstream kinases in the AMP-activated protein kinase cascade. J Biol 2(4):28. https:// doi.org/10.1186/1475-4924-2-28

23. Nakanishi A, Hatano N, Fujiwara Y, Sha'ri A, Takabatake S, Akano $\mathrm{H}$ et al (2017) AMP-activated protein kinase-mediated feedback phosphorylation controls the $\left.\mathrm{Ca}^{2+}\right)$ /calmodulin $(\mathrm{CaM})$ dependence of $\mathrm{Ca}\left({ }^{2+}\right) / \mathrm{CaM}$-dependent protein kinase kinase $\beta$. Journal Biol Chem 292(48):19804-19813

24. Chen L, Wang J, You Q, He S, Meng Q, Gao J et al (2018) Activating AMPK to restore tight junction assembly in intestinal epithelium and to attenuate experimental colitis by metformin. Front Phamacol 9:761. https://doi.org/10.3389/fphar.2018.00761

25. Peng L, Li ZR, Green RS, Holzman IR, Lin J (2009) Butyrate enhances the intestinal barrier by facilitating tight junction assembly via activation of AMP-activated protein kinase in Caco-2 cell monolayers. J. Nutr. 139(9):1619-1625

26. Koppelmann T, Pollak Y, Mogilner J, Bejar J, Coran AG, Sukhotnik I (2012) Dietary L-arginine supplementation reduces methotrexate-induced intestinal mucosal injury in rat. BMC Gastroenterol 12:41. https://doi.org/10.1186/1471-230X-12-41

27. Costa KA, Soares ADN, Wanner SP, Santos RD, Fernandes SO, Martins Fdos S et al (2013) L-Arginine supplementation prevents increases in intestinal permeability and bacterial translocation in male swiss mice subjected to physical exercise under environmental heat stress. J Nutr 144(2):218-223

28. Chen C, Xia R, Chen H, He YH (2018) TBtools, a Toolkit for Biologists integrating various HTS-data handling tools with a user-friendly interface. J BioRxiv. https://doi.org/10.1101/289660

29. Kong S, Zhang YH, Zhang W (2018) Regulation of intestinal epithelial cells properties and functions by amino acids. Biomed Res Int 2018:2819154. https://doi.org/10.1155/2018/2819154

30. Landriscina M, Amoroso MR, Piscazzi A, Esposito F (2010) Heat shock proteins, cell survival and drug resistance: the mitochondrial chaperone TRAP1, a potential novel target for ovarian cancer therapy. Gynecol Oncol 117(2):177-182

31. Wu G, Bazer FW, Davis TA, Kim SW, Li P, Marc Rhoads J et al (2009) Arginine metabolism and nutrition in growth, health and disease. Amino Acids 37(1):153-168
32. Yi G, Li L, Luo M, He X, Zou Z, Gu Z et al (2017) Heat stress induces intestinal injury through lysosome- and mitochondria-dependent pathway in vivo and in vitro. Oncotarget 8(25):40741-40755

33. Fleming SD, Starnes BW, Kiang JG, Stojadinovic A, Tsokos GC, Shea-Donohue T (2002) Heat stress protection against mesenteric I/R-induced alterations in intestinal mucosa in rats. J Appl Physiol 92(6):2600-2607

34. Hall DM, Buettner GR, Oberley LW, Xu L, Matthes RD, Gisolfi CV (2001) Mechanisms of circulatory and intestinal barrier dysfunction during whole body hyperthermia. Am J Physiol Heart Circ Physiol 280(2):H509-H521

35. Yang PC, He SH, Zheng PY (2007) Investigation into the signal transduction pathway via which heat stress impairs intestinal epithelial barrier function. J Gastroenterol Hepatol 22(11):1823-1831

36. Pan L, Zhao PF, Ma XK, Shang QH, Xu YT, Long SF et al (2017) Probiotic supplementation protects weaned pigs against enterotoxigenic Escherichia coli K88 challenge and improves performance similar to antibiotics. J Anim Sci 95(6):2627-2639

37. Chen JL, Zheng P, Zhang C, Yu B, He J, Yu J et al (2017) Benzoic acid beneficially affects growth performance of weaned pigs which was associated with changes in gut bacterial populations, morphology indices and growth factor gene expression. J Anim Physiol Anim Nutr 101(6):1137-1146

38. Zhu M-J, Sun X, Du M (2018) AMPK in regulation of apical junctions and barrier function of intestinal epithelium. Tissue Barriers 6(2):1-13

39. Wang T, Yu Q, Chen J, Deng B, Qian L, Le Y (2010) PP2A mediated AMPK inhibition promotes HSP70 expression in heat shock response. PLoS ONE 5(10):e13096. https://doi.org/10.1371/journ al.pone. 0013096

40. Varasteh S, Braber S, Kraneveld AD, Garssen J, Fink-Gremmels J (2018) L-Arginine supplementation prevents intestinal epithelial barrier breakdown under heat stress conditions by promoting nitric oxide synthesis. Nutr Res. 57:45-55

41. Nalli AD, Kumar DP, Mahavadi S, Al-Shboul O, Alkahtani R, Kuemmerle JF et al (2014) Hypercontractility of intestinal longitudinal smooth muscle induced by cytokines is mediated by the nuclear factor- $\mathrm{\kappa B} / \mathrm{AMP}$-activated kinase/myosin light chain kinase pathway. J Pharmacol Exp Ther 350(1):89-98

42. Sun X, Yang Q, Rogers CJ, Du M, Zhu MJ (2017) AMPK improves gut epithelial differentiation and barrier function via regulating Cdx2 expression. Cell Death Differ 24(5):819-831

43. Kahn BB, Alquier T, Carling D, Hardie DG (2005) AMP-activated protein kinase: ancient energy gauge provides clues to modern understanding of metabolism. Cell Metab 1(1):15-25

44. Zheng B, Cantley LC (2007) Regulation of epithelial tight junction assembly and disassembly by AMP-activated protein kinase. Proc Nati Acad Sci USA. 104(3):819-822

Publisher's Note Springer Nature remains neutral with regard to jurisdictional claims in published maps and institutional affiliations. 


\section{Affiliations}

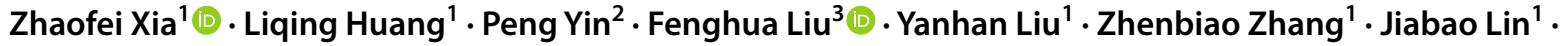
Wenshu Zou ${ }^{3}$. Chunxiao $\mathrm{Li}^{3}$

Liqing Huang

libbyhuang@cau.edu.cn

Peng Yin

Yinp@im.ac.cn

Fenghua Liu

liufenghua1209@126.com

Yanhan Liu

liuyanhan@cau.edu.cn

Zhenbiao Zhang

zbzhang2017@cau.edu.cn

Jiabao Lin

B20163050375@cau.edu.cn
Wenshu Zou

zouwenshu080603@163.com

Chunxiao Li

lcx@bua.edu.cn

1 Department of Veterinary Medicine, College of Veterinary Medicine, China Agricultural University, No. 2 Yuan Ming Yuan Western Road, Hai Dian District, Beijing 100193, China

2 Institute of Microbiology, Chinese Academy of Sciences, Beijing 100101, China

3 College of Animal Science and Technology, Beijing Agricultural University, Beijing 102206, China 